\title{
Na Literatura Internacional, como Está Sendo Pesquisado o Papel de Gerente no Desenvolvi- mento das Capacidades Dinâmicas?
}

\section{In the International Literature, how the Manager's Role Is Being Researched in the Develop- ment of Dynamic Capabilities?}

\author{
Gibson Meira Oliveira \\ Universidade Federal da Paraíba - UFPB - Brasil \\ gibson.meira@gmail.com \\ ORCID: 0000-0002-0835-7499
}

Submetido em 25/02/2020; Aprovado em 09/04/2020.

\begin{abstract}
Resumo
Objetivo: este texto tem como objetivo analisar como está sendo pesquisado, nos artigos publicados internacionalmente, o papel de gerentes no processo de desenvolvimento de Capacidades Dinâmicas. Metodologia: foi realizada uma Revisão Sistemática da Literatura dos artigos disponíveis no maior repositório de trabalhos acadêmicos internacional na área de Administração, a Web of Science. Por meio de um processo de busca e filtragem, foram identificados diversos trabalhos científicos, os quais passaram por um procedimento de leitura e análise minuciosa, possibilitando a compreensão do propósito dos trabalhos, sua natureza, estratégias de pesquisa, métodos e outras informações relevantes. Constatações: foi possível constatar que existem poucos artigos que abordam o papel do gerente na literatura sobre Capacidades Dinâmicas. Após a proposição do conceito de Capacidade Gerencial Dinâmica e seus atributos, por Adner e Helfat (2003), novas pesquisas foram desenvolvidas, porém, até 2019 não foi possível observar estudos que aprofundassem de fato o construto em sua completude. Contribuições: este trabalho contribui para o campo acadêmico com informações relevantes à pesquisa sobre Capacidades Dinâmicas e, de uma maneira mais ampla, sobre Estratégia Organizacional, além de permitir a criação de uma agenda de pesquisas futuras. Em nível prático, a identificação de trabalhos empíricos sobre a temática pode auxiliar no desenvolvimento de estratégias organizacionais alinhadas às perspectivas das Capacidades Dinâmicas e suas aplicações em ambientes organizacionais e interorganizacionais.
\end{abstract}

Palavras-Chave: Capacidades Dinâmicas, Capacidades Gerenciais Dinâmicas, Gerente

\begin{abstract}
Objective: this article aims to analyze how are being researched the role of managers in the process of developing Dynamic Capabilities in articles published internationally. Methodology: it was made a Systematic Literature Review of the articles available in the largest international repository of academic papers in the field of Business, the Web of Science. Through a search and filtering process, several scientific works were identified, which underwent a thorough reading and analysis procedure, enabling the understanding of the purpose of the works, their nature, research strategies, methods and other relevant information. Findings: it was found that there are few articles that address the role of the manager in the literature on Dynamic Capabilities. After the proposal of the Dynamic Managerial Capabilities and its attributes, by Adner and Helfat (2003), new researches were developed, however, until 2019 it was not possible to observe studies that really deepened the construct. Contributions: this work contributes to the academic field by condensing information relevant to research on Dynamic Capabilities and, more broadly, on Organizational Strategy, in addition to allowing the creation of an agenda for future research. In practical terms, through the identification of empirical works that become references to be applied in the field, it allows the development of organizational strategies aligned with the perspectives of Dynamic Capabilities.
\end{abstract}

Keywords: Dynamic Capabilities, Dynamic Managerial Capabilities, Manager 


\section{Introdução}

De acordo com Teece, Pisano e Shuen (1997), o construto Capacidade Dinâmica é a capacidade de se integrarem, se construírem, interna e externamente, competências para atender aos ambientes de rápidas mudanças. Tal perspectiva (Capacidade Dinâmica) tem por objetivo criar um marco teórico em relação às vigentes teorias em estratégia empresarial, estudando-se nuances ainda não contempladas pela Visão Baseada em Recursos de Penrose (1959) e Wernerfelt (1984).

A Visão Baseada em Recursos amplia o escopo para uma perspectiva mais abrangente, apesar de ser largamente estudada por teóricos ao longo dos anos. A teoria das Capacidades Dinâmicas ganhou robustez por meio das diversas discussões teóricas e também a partir de estudos empíricos os quais foram validados e comprovados. Desde seu surgimento em 1997 até os dias atuais, milhares de artigos publicados sobre esse assunto podem ser encontrados nas principais bases indexadoras.

Nas produções científicas encontradas, em uma rápida busca realizada na Web of Science, observase que, atualmente, os estudos relacionados às Capacidades Dinâmicas abrangem diversas áreas do conhecimento, de maneira proeminente na Administração, mas também alcança as Ciências da Computação, Engenharia, Gerenciamento de Operações, Tecnológica e outros.

Apesar da grande abrangência e representatividade na Administração, numa análise mais cuidadosa e profunda de artigos envolvendo essas capacidades, percebe-se que o construto tem por objetivo compreender a dinamicidade do mercado e como a empresa se adequa a ele. Ao passo em que os pesquisadores se aprofundam na pesquisa e no conhecimento, surgem, porém, divergências quanto à interpretação do desenvolvimento da teoria, assim como novas perspectivas e caminhos de interpretação.

Um exemplo dessas divergências que envolvem a discussão relacionada à natureza das Capacidades Dinâmicas nos é revelado em dois estudos: a) os de Teece et al. (1997), que adotam uma perspectiva de que as Capacidades Dinâmicas são definidas por habilidades latentes e competências, e; b) os de Zollo e Winter (2000), que a definem com base nos processos e rotinas. Da mesma forma, surgiram teorias complementares que abrangem perspectivas não contempladas de maneira explícita no construto.

Uma dessas perspectivas incorpora o papel do gerente enquanto parte da empresa e no desenvolvimento das Capacidades Dinâmicas. Tal abordagem é apontada por Adner e Helfat (2003), possibilitando o surgimento de um novo construto denominado Capacidades Gerenciais Dinâmicas, compreendida como a capacidade de o gerente construir, integrar e reconfigurar os recursos e competências da organização.

O conceito de Capacidade Gerencial Dinâmica está analogamente relacionado ao que foi proposto por Teece et al. (1997) em relação Às Capacidades Dinâmicas. Vale esclarecer que o foco deste nosso estudo está mais relacionado com o desenvolvimento de pesquisas orientadas para a compreensão do papel gerencial no desenvolvimento das Capacidades Dinâmicas, tornando-se uma perspectiva paralela ou complementar a sua natureza.

Apesar da existência desse construto voltado para o papel do gerente, ainda restam dúvidas quanto à sua abordagem. Surge, portanto, o questionamento: Nos artigos publicados sobre Capacidades Dinâmicas, como está sendo pesquisado o papel do gerente no processo, sendo ele parte da organização?

Para compreender como essas pesquisas tratam o papel do gerente nas abordagens teóricas relacionadas às Capacidades Dinâmicas, faz-se necessário realizar um levantamento bibliográfico sobre o tema, para verificar se esse agente é referenciado ou não nas pesquisas relacionadas ao tema, assim como para entender a trajetória de desenvolvimento do construto proposto por Adner e Helfat (2003).

A realização de um levantamento bibliográfico na principal base indexadora de trabalhos científicos da área de Administração, a Web of Science, auxiliará na obtenção de respostas, permitindo, principalmente, compreender o desenvolvimento da temática em relação ao papel do gerente no desenvolvimento estratégico das Capacidades Dinâmicas. A Revisão Sistemática da Literatura sobre o tema tem como principais contribuições: apresentar uma análise dos trabalhos cuja abordagem contenha um direcionamento para compreensão do papel do gerente no desenvolvimento de capacidades dinâmicas; identificar gaps teóricos existentes nessa temática; e apresentar direções de pesquisas futuras que possam complementar o conhecimento relacionado ao construto em questão.

\section{Referencial Teórico}

De acordo com Teece et al. (1997), as Capacidades Dinâmicas referem-se a: 1)novas formas de 
obter vantagens competitivas, com ênfase na dinâmica de renovação de competências, de forma congruente aos ambientes de mudanças mercadológicas, e; 2) a capacidade estratégica de gestão para adaptar, integrar e reconfigurar a organização. Entende-se, por esta perspectiva, que os autores adotam uma abordagem muito mais voltada às habilidades e competências internas, para atuar frente a questões externas à organização.

Outro olhar em relação ao construto é a de Zollo e Winter (2002). Estes compreendem que o desenvolvimento das capacidades dinâmicas envolve um mecanismo de aprendizagem relacionado com atributos organizacionais, tais como: a experiência acumulada, os conhecimentos articulados e os codificados, que, por sua vez, levam a capacidades dinâmicas que auxiliam nos processos de pesquisa e desenvolvimento, reestruturação e reengenharia e de integração do conhecimento. Em conjunto, consequentemente, aprimoram as rotinas operacionais. Trata-se de uma visão baseada em processos e rotinas.

As teorias das Capacidades Dinâmicas, em sua essência, relacionam-se com a empresa como um todo, adotando uma perspectiva estratégica organizacional. Nos estudos mais conhecidos e seminais da teoria (Teece, Pisano, \& Shuen, 1997; Eisenhardt \& Martin, 2000; Zollo \& Winter, 2002; Teece, 2007; Augier \& Teece, 2009), não existe uma ênfase nas pessoas, mas no desenvolvimento organizacional.

Essas abordagens caminham paralelamente, e novas perspectivas foram sendo incrementadas às teorias relacionadas a este assunto. Teece (2007), por exemplo, incorpora o nível individual, ao afirmar que as capacidades dinâmicas envolvem processos de detecção, aproveitamento de oportunidades, compreendendo com isso a transformação e ou reconfiguração das capacidades, objetivando construir vantagens competitivas para a organização. E mais recentemente, entende-se tal objeto como a capacidade da empresa de gerenciar alianças, aprender, integrar e reconfigurar a base de recursos para atender às mudanças nas condições de negócios (Singh \& Rao, 2016).

Tempos atrás, autores como Adner e Helfat (2003) incorporam o papel do gerente/gestor no desenvolvimento das capacidades dinâmicas e indicam a necessidade de estudar o tema sob uma perspectiva própria, surgindo, assim, uma teoria complementar e paralela àquela já estabelecida, denominada de Capacidades Gerenciais Dinâmicas.

A Capacidade Gerencial Dinâmica é a habilidade do gerente de construir, integrar e reconfigurar os recursos e competências da organização diante de um mercado em rápidas mudanças (Adner \& Helfat, 2003; Helfat \& Martin, 2015). Assim sendo, o gerente é figura representativa no processo de gestão de recursos, competências e habilidades internas da organização, objetivando adequá-la aos mercados, cujas mudanças acontecem de maneira rápida.

Os autores identificaram três atributos dos gerentes que auxiliam no desenvolvimento das capacidades gerenciais dinâmicas, são eles: capital social gerencial, capital humano gerencial e cognição gerencial, apresentados a seguir:

- capital social gerencial: abrange as redes de relacionamentos sociais existentes por parte do gerente. Assim, quanto mais influência social, acesso a informações ou contatos, maior será o capital social desse agente.

- capital humano gerencial: diz respeito às capacidades, competências e formações que o gerente possui - seus conhecimentos específicos, formação acadêmica, experiências prévias obtidas por meio do desenvolvimento da função ou outras que complementem seu "know how".

- cognição gerencial: está relacionada à forma como o gerente pensa, toma decisões, sendo este fator fortemente influenciado pela natureza pessoal, suas experiências prévias sobre determinados assuntos, de fato, o modelo mental de tomada de decisão, é próprio, logo variável de pessoa para pessoa.

Esses são atributos propostos pelos autores, por meio dos quais os gerentes conseguem, a partir de competências e recursos internos e externos, possibilitar que a organização desenvolva capacidades dinâmicas, para atender estrategicamente ao mercado.

\section{Metodologia}

O presente estudo adota uma perspectiva teórica; o método adotado é a Revisão Sistemática da Literatura (RSL). Quanto à natureza, considera-se quantitativa, porque utiliza um método para avaliar e quantificar a produção científica em determinado lapso temporal (ARAÚJO, 2006); e, qualitativa, já que 
é feita por meio da análise dos conteúdos, uma vez que possibilita realizar um levantamento teórico dos achados apresentados nos trabalhos analisados, agregando maior conhecimento e desenvolvendo um entendimento sólido sobre o tema (Conforto, Amaral, \& Silva, 2011).

Considerando os diversos tipos de RSLs, cada qual com características e finalidades próprias, esta pesquisa caracteriza-se como um mapeamento, cujo objetivo é classificar os estudos e compreender o desenvolvimento de uma determinada temática (Kitchenham et al., 2010). Classifica-se como uma revisão descritiva (King \& He, 2005), utilizando-se de métodos estatísticos mais simplificados (descritivos) para apresentar frequências e percentuais, expondo o panorama geral dos temas abordados.

Conforto, Amaral e Silva (2011) propõem um método de desenvolvimento desse tipo de pesquisa, o qual se baseia em três fases (entrada, processamento e saída). A primeira estabelece os objetivos e o problema, um breve referencial; nesta fase são definidos critérios de inclusão e exclusão, os métodos e ferramentas utilizados. A segunda fase corresponde à busca nos indexadores, analisando previamente o resultado e a adequação aos objetivos estipulados. Por fim, na terceira fase, é elaborado um documento final que sintetiza os achados. Considerando a aceitação e a eficiência do método, optou-se pela sua utilização para o levantamento sobre a produção científica envolvendo o tema Capacidades Gerenciais Dinâmicas.

O levantamento bibliográfico foi realizado na base indexadora da Web of Science, no período de 1997 (quando surgem os primeiros trabalhos relacionados ao tema) até 2019. A escolha da base de pesquisa para o presente estudo levou em consideração que a Web of Science possui a maior base de dados de trabalhos acadêmicos publicados, além de indexar o maior número de periódicos de maior relevância no universo acadêmico da Administração. Buscas distintas foram realizadas para os temas Capacidades Dinâmicas e Capacidades Gerenciais Dinâmicas.

\subsection{Critérios de busca}

Na primeira busca realizada, não foram inicialmente utilizados filtros, para que se tivesse uma dimensão apropriada de como o termo se comportava na base indexadora do Web of Science. Assim, o termo utilizado foi "Dynamic Capability". Esta pesquisa resultou inicialmente num total de 1.003 trabalhos, porém, destes, apenas 646 eram artigos científicos; os demais dividiam-se em: papéis de procedimentos, revisões, capítulo de livros, material editorial, notas e outros. Sobre esse resultado, realizou-se uma filtragem, restringindo-se os achados apenas aos trabalhos da área de Administração, restando, portanto, 363 artigos.

Levando-se em conta que o objetivo desta investigação é analisar como está sendo pesquisada a figura do gerente na literatura relacionada às Capacidades Dinâmicas, nos artigos publicados internacionalmente, foi realizada a leitura dos títulos e resumos para identificar a perspectiva ou foco no papel do gerente naqueles trabalhos. Essa leitura resultou em 31 artigos selecionados para avaliação.

Uma vez realizada essa seleção prévia, os 31 artigos foram lidos na íntegra para ratificar que realmente abordavam o papel do gerente/gestor no desenvolvimento das Capacidades Dinâmicas. Após a leitura, apenas 12 trabalhos atendiam aos requisitos de busca, com exceção de 01 deles que não foi possível analisar na íntegra e acabou sendo excluído da análise.

Uma segunda busca foi realizada na mesma base indexadora, porém utilizando-se o termo "Dynamic Managerial Capability". Não foram utilizados filtros. Os resultados revelaram 46 trabalhos, dos quais 44, embora relacionados com a área de Administração, também se adequam preeminentemente à Engenharia, Ciências da Computação e outras áreas. Do total de 46, 43 são artigos e os 03 outros classificaram-se como revisões.

Por ser um subtema específico das Capacidades Dinâmicas, com foco de análise direcionado ao gerente, a única filtragem realizada foi para separar os artigos relacionados à área de Administração, restando os 43 acima citados. Eles foram lidos na íntegra, sendo descartados apenas 04 por não estarem vinculados ao construto (acredita-se em falha no mecanismo de busca da base), restando, assim, 39 trabalhos.

\subsection{Análise}

A análise dos artigos anteriormente filtrados ocorreu por meio de uma leitura integral dos trabalhos pré-selecionados. Realizou-se uma leitura sistemática em que se teve o cuidado de se buscar e se identificar a relação dos trabalhos ao tema Capacidades Dinâmicas. Se constatado o alinhamento ao 
tema, passava-se a identificar a abordagem da perspectiva do gerente e se a pesquisa continha, de fato, um direcionamento voltado ao estudo do papel desse agente.

Dessa forma, após a análise dos resultados das pesquisas realizadas e de acordo com a quantidade de artigos com foco no gerente que abordam o desenvolvimento das Capacidades Dinâmicas, foram identificados 51 artigos sobre Capacidades Dinâmicas e Capacidades Gerenciais Dinâmicas, publicados em 40 revistas internacionais. Um relevante fator é o crescente número de publicações nos últimos quatro anos, como pode ser observado no Gráfico 1.

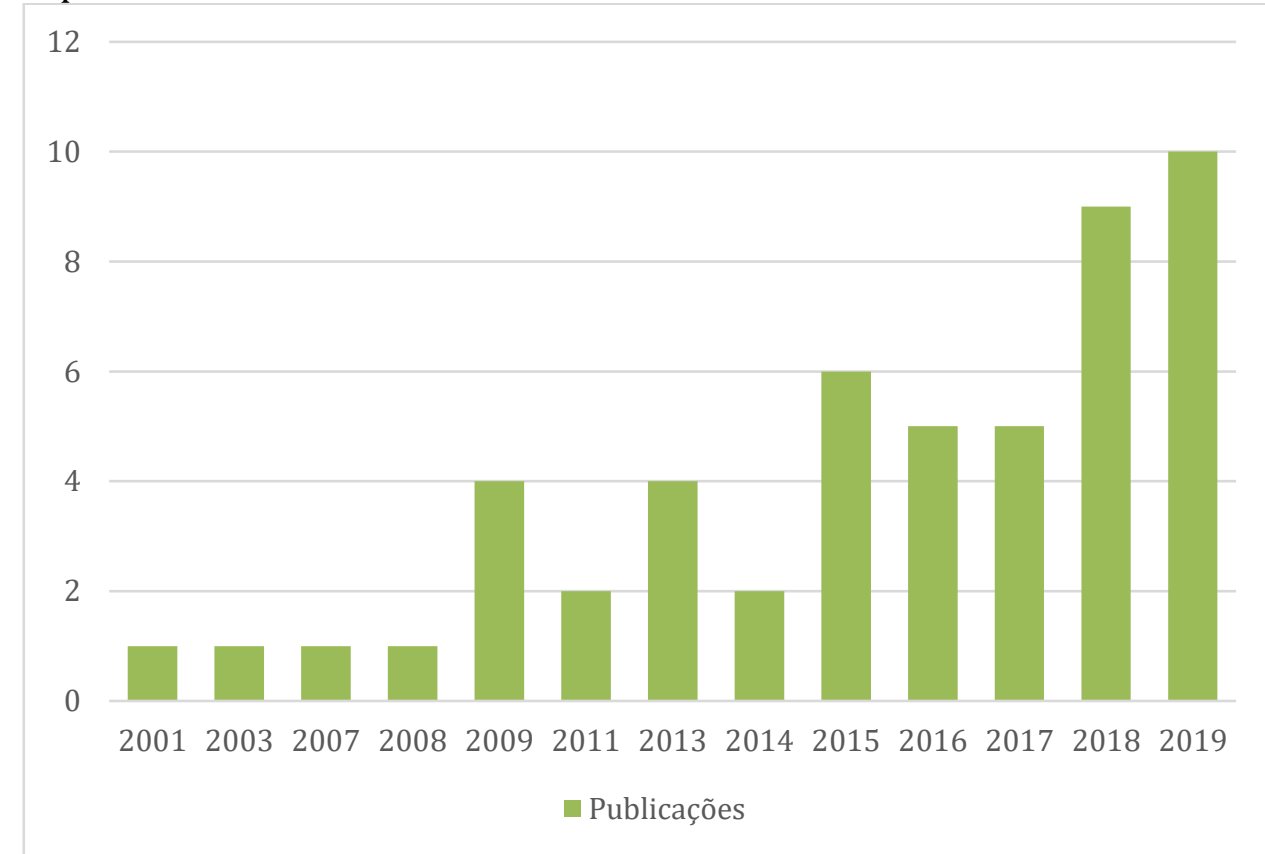

Gráfico 1. Artigos publicados por ano

Fonte: Elaborado pelo autor.

Apesar de o gráfico mostrar que as publicações sobre o tema tiveram início em 2001, quando foram publicados os primeiros artigos, segundo busca no Web of Science, é interessante destacar que o termo Capacidades Dinâmicas foi inicialmente proposto por Teece et al. (1997).

A Tabela 1 apresenta os principais periódicos, bem como o número de publicações identificadas para os trabalhos selecionados. Observa-se que o Strategic Management Journal possui o maior número de publicações, tornando-se o mais representativo da área. Outros, como Organization Science e Industrial Marketing Management, apresentam três publicações sobre o tema no período analisado.

Tabela 1. Periódicos com mais publicações sobre Capacidades Dinâmicas e Capacidades Gerencias Dinâmicas

\begin{tabular}{|c|l|c|}
\hline Ranque & \multicolumn{1}{|c|}{ Periódico } & Quantidade \\
\hline $\mathbf{1}$ & STRATEGIC MANAGEMENT JOURNAL & 7 \\
\hline $\mathbf{2}$ & ORGANIZATION SCIENCE & 3 \\
\hline $\mathbf{3}$ & INDUSTRIAL MARKETING MANAGEMENT & 3 \\
\hline $\mathbf{4}$ & JOURNAL OF ORGANIZATIONAL CHANGE MANAGEMENT & 2 \\
\hline $\mathbf{5}$ & JOURNAL OF MANAGEMENT AND GOVERNANCE & 2 \\
\hline $\mathbf{r}$ & OUTROS & 34 \\
\hline
\end{tabular}

Nota. Fonte: Elaborado pelo autor

No Tabela 2, estão listados os autores/coautores que apresentam maior frequência de publicações sobre Capacidades Dinâmicas com foco no papel do gerente. Percebe-se que a Constance E. Helfat, que é a precursora do construto Capacidades Gerenciais Dinâmicas, juntamente com o Jeffrey A. Martin, publicaram três artigos sobre o tema. Dessa forma, o conhecimento dos trabalhos desses autores torna-se fundamental para o delineamento de futuras pesquisas e a compreensão do tema como um todo. 
Tabela 2 - Autores e publicações sobre Capacidades Dinâmicas e Capacidades Gerenciais Dinâmicas

\begin{tabular}{|l|c|}
\hline \multicolumn{1}{|c|}{ Autor/Coautor } & Qtd. Publicações \\
\hline Constance E. Helfat & 3 \\
\hline Jeffrey A. Martin & 3 \\
\hline Carl Åberg & 2 \\
\hline Farhan Khan & 2 \\
\hline Fouzia Atlas & 2 \\
\hline Kashif Ullah Khan & 2 \\
\hline Llorenc Bagur-Femenias & 2 \\
\hline Maria del Mar Alonso-Almeida & 2 \\
\hline Marian Buil-Fabrega & 2 \\
\hline Md Imtiaz Mostafiz & 2 \\
\hline Murali Sambasivan & 2 \\
\hline See Kwong Goh & 2 \\
\hline Wei Shen & 2 \\
\hline Zhang Xuehe & 2 \\
\hline
\end{tabular}

Nota. Fonte: Elaborado pelo autor

\section{Análise dos Resultados}

Após o mapeamento dos trabalhos e o processo de análise, descritos na metodologia, nesta seção serão apresentados os principais achados e, também, uma análise e discussão dos trabalhos, permitindo realizar e fomentar uma reflexão sobre a abordagem do papel do gerente nas pesquisas sobre Capacidades Dinâmicas.

Em um primeiro momento, serão apresentadas a análise e discussão em relação aos trabalhos sobre Capacidades Dinâmicas e como a figura do gerente é abordada. Em sequência, discute-se o construto Capacidades Gerenciais Dinâmicas, seus aspectos, características e importância. Por fim, caracteriza-se o cenário da pesquisa sobre o tema, o que reflete o atual momento e perspectivas futuras.

\subsection{Capacidades dinâmicas e a perspectiva do gerente}

Após o refinamento da pesquisa realizada, a leitura dos resumos e trabalhos completos, o resultado final indica 11 artigos que abordam o papel do gerente no processo de desenvolvimento das Capacidades Dinâmicas. Na Tabela 3, estão elencados os trabalhos citados.

Tabela 3 - Trabalhos sobre capacidades dinâmica e a perspectiva gerencial

Autor

Título

Ano

\begin{tabular}{|l|l|c|}
\hline \multicolumn{1}{|c|}{ Autor } & \multicolumn{1}{c|}{ Título } & Ano \\
\hline Carpenter, Sanders, \& Gregersen & $\begin{array}{l}\text { Bundling human capital with organizational context: The impact of inter- } \\
\text { national assignment experience on multinational firm performance and } \\
\text { CEO pay }\end{array}$ & 2001 \\
\hline Witcher, Chau, \& Harding & $\begin{array}{l}\text { Dynamic capabilities: top executive audits and hoshin kanri at Nissan } \\
\text { South Africa }\end{array}$ & 2008 \\
\hline Narayanan, Colwell, \& Douglas & $\begin{array}{l}\text { Building Organizational and Scientific Platforms in the Pharmaceutical In- } \\
\text { dustry: A Process Perspective on the Development of Dynamic Capabilities }\end{array}$ & 2009 \\
\hline O'Reilly \& Tushman & $\begin{array}{l}\text { Organizational Ambidexterity in Action: How MANAGERS EXPLORE AND } \\
\text { EXPLOIT }\end{array}$ & 2011 \\
\hline Fernandez-Mesa, Iborra, \& Safon & CEO-TMT interaction: do tenure and age affect ambidexterity dynamism? & 2013 \\
\hline Lecler \& Kinghorn & Dynamic capabilities, expert and entrepreneurial learning \\
\hline Chang, Bai, \& Li & $\begin{array}{l}\text { The influence of leadership on product and process innovations in China: } \\
\text { The contingent role of knowledge acquisition capability }\end{array}$ & 2014 \\
\hline Ansari, Barati, \& Sharabiani & $\begin{array}{l}\text { The role of dynamic capability in intellectual capital and innovative perfor- } \\
\text { mance }\end{array}$ & 2016 \\
\hline Akrofi & $\begin{array}{l}\text { Evaluating the effects of executive learning and development on organisa- } \\
\text { tional performance: implications for developing senior manager and exec- } \\
\text { utive capabilities }\end{array}$ & 2016 \\
\hline Singh \& Rao & Effect of intellectual capital on dynamic capabilities \\
\hline Ramachandran & $\begin{array}{l}\text { Triggering absorptive capacity in organizations: CEO succession as a } \\
\text { knowledge enabler }\end{array}$ & 2016 \\
\hline
\end{tabular}

Notas. Fonte: Elaborado pelo autor 
Carpenter, Sanders e Gregersen (2001) realizaram uma pesquisa empírica na qual os autores investigam a influência de experiências internacionais no processo de desenvolvimento das Capacidades Dinâmicas. Os resultados do estudo revelam uma relação positiva, ou seja, quanto maior as experiências internacionais dos gerentes, melhor será o desempenho, relacionando-se diretamente com o capital humano desse profissional.

Já Witcher, Chau e Harding (2008) investigaram como ocorre o processo de auditoria dos gerentes de alto posicionamento no setor de desenvolvimento estratégico e operacional. Como resultado, eles compreendem que há uma maior intensidade no comportamento empreendedor e na flexibilidade em empresas que notoriamente desenvolvem fortes capacidades dinâmicas, não apenas por questões econômicas circunstanciais, mas também pela competência da organização em gerenciar as mudanças de uma forma efetiva.

Compreender quais ações os gerentes podem empreender para o desenvolvimento das capacidades dinâmicas é o tema abordado pelos autores Narayanan, Colwell e Douglas (2009), visando a delimitar o papel desses profissionais. Trata-se de artigo empírico em que os autores sugerem alguns aspectos considerados relevantes no processo de desenvolvimento das capacidades dinâmicas e da organização. Estes autores chamam a atenção, porém, para a incapacidade de "importar" competências do ambiente externo.

Aspectos como ambidestria são trabalhados pelos autores O'Reilly e Tushman (2011), com o objetivo de analisar como a liderança possui um complexo dever de orquestrar simultaneamente o exploration e exploitation. Empiricamente, são testadas cinco proposições relacionadas com intenções estratégicas, visão e valores, equipe, arquitetura organizacional e capacidade de liderança como fatores de influências no processo de exploração interna e externa. Por fim, entende-se que as ações, o comportamento e a forma como as decisões são tomadas permitem a organização desenvolver capacidades dinâmicas.

Na base de dados do Web of Science, só foi possível identificar o resumo do trabalho de FernandezMesa, Iborra e Safon (2013). Uma análise do resumo sugere que os autores trabalham com a perspectiva do $C E O$, porém não foi possível obter conhecimento mais aprofundado, devido à inacessibilidade ao trabalho.

Lecler e Kinghorn (2014) exploram, em seu artigo, como os gerentes percebem e aproveitam oportunidades de aprendizagem, e buscaram ampliar a compreensão da teoria da cognição gerencial. Os dados levaram os autores a concluírem que padrões comuns de aprendizagem estão relacionados a dois aspectos: experiência e empreendedorismo. Já o reconhecimento de oportunidades provém de inovações tecnológicas e organizacionais. Com isso, propõem um modelo que envolve quatro diferentes perspectivas: aperfeiçoamento especializado, alinhamento por especialistas, modelagem empreendedora e configuração empreendedora.

Para compreender e melhor distinguir as diferenças entre inovação de processos e produtos, Chang, Bai e Li (2015) buscaram avaliar o impacto efetivo da liderança transacional e transformacional carismática nos dois tipos de inovação. Eles comprovaram o impacto de ambos os tipos de liderança nos dois tipos das referidas inovações. Complementarmente, concluíram que a capacidade de aquisição de conhecimento pode melhor influenciar todo o processo.

Ansari, Barati e Sharabiani (2016) também buscaram compreender a influência do Capital Intelectual no processo de inovação, considerando as Capacidades Dinâmicas como variável moderadora. Eles concluem que o gerente não deve, principalmente, dar atenção às mudanças de mercado, mas sim aos recursos humanos e ao capital intelectual, enfatizando os capitais estruturais, organizacionais e relacionais. Essa mesma ênfase deve se dar também em relação à identificação, percepção e reconfiguração dos recursos diante das oportunidades.

Seguindo a mesma linha, Singh e Rao (2016) realizaram um estudo empírico em instituições financeiras do setor público na Índia, em que gerentes são estudados para compreender os efeitos do capital intelectual (para os autores, capital social e organizacional) nas capacidades dinâmicas (aprendizagem, integração, reconfiguração e aliança gerencial). 0 estudo revelou que há um forte efeito das dimensões do capital intelectual nas capacidades dinâmicas, porém ressalta-se que o capital organizacional possui um efeito negativo na reconfiguração e aliança de gerentes, fato que precisa ser melhor investigado. 
Por fim, o último e mais recente estudo, o de Ramachandran (2018), busca compreender teoricamente se o $C E O$ é capaz de deixar conhecimento em sua sucessão. Para embasar sua pesquisa, Ramachandran (2018) utiliza-se das dimensões da capacidade absortiva (Zahra \& George, 2002) e conclui que, a depender do tipo de sucessão, combinando com a possível rotatividade do executivo, ocorre uma reorientação na experiência e nos conhecimentos da alta gerência.

Utilizando-se a estatística descritiva e analisando-se os artigos sobre Capacidades Dinâmicas, observou-se que 58,33\% dos trabalhos são quantitativos e $41,66 \%$ são qualitativos, havendo, assim, uma predominância de estudos que utilizam ferramentais estatísticos, o que está muito relacionado com o tipo de método de pesquisa adotado, como é apresentado na Tabela 4.

Tabela 4 - Métodos de Pesquisa utilizados nos estudos sobre Capacidades Dinâmicas

\begin{tabular}{|l|c|c|}
\hline Método de Pesquisa & Frequência & Percentual \\
\hline Survey & 7 & $58,33 \%$ \\
\hline Estudo de caso & 4 & $33,33 \%$ \\
\hline Revisão da Literatura & 1 & $8,33 \%$ \\
\hline Total & $\mathbf{1 2}$ & $\mathbf{1 0 0 \%}$ \\
\hline
\end{tabular}

Notas. Fonte: Elaborado pelo autor

Há uma predominância de trabalhos cujo método é o Survey, ou seja, há uma tendência dos pesquisadores em utilizar métodos estatísticos para dar suporte à análise, além do maior volume de informações que precisam ser processadas quando se considera um estudo do tipo empírico, como é possível observar na Tabela 5.

Tabela 5 - Tipos de Pesquisas utilizados nos estudos sobre Capacidades Dinâmicas

\begin{tabular}{|l|c|c|}
\hline Tipos de Pesquisa & Frequência & Percentual \\
\hline Teórico - Empírico & 6 & $50,00 \%$ \\
\hline Empírico & 5 & $41,66 \%$ \\
\hline Teórico & 1 & $8,33 \%$ \\
\hline Total & $\mathbf{1 2}$ & $\mathbf{1 0 0 \%}$ \\
\hline
\end{tabular}

Notas. Fonte: Elaborado pelo autor

Mais de 90\% dos estudos são de natureza empírica, o que representa, de certa forma, maturidade da temática e, com isso, a necessidade de os pesquisadores buscarem comprovações por meio de testes e pesquisas empíricas. Também é possível identificar que mais da metade dos trabalhos analisados possuem proposições teóricas ao construto Capacidades Dinâmicas (considerando-se as vertentes dessas pesquisas), indicando que ainda existem gaps teóricos passíveis de novos estudos.

Percebe-se, porém, que os diversos estudos refletem o papel do gerente no processo de desenvolvimento das capacidades dinâmicas. Alguns trabalhos deixam clara sua intenção e relação com a teoria pesquisada, outros não deixam explícito que pretendem complementar o conhecimento da temática. Após a caracterização dos estudos envolvendo capacidades dinâmicas, é possível, contudo, discutir com maior propriedade e comparar com mais segurança o papel do gerente em ambas as perspectivas. $\mathrm{Na}$ próxima seção, apresenta-se a análise de artigos que utilizam a expressão Capacidade Gerencial Dinâmica.

\subsection{Capacidades gerenciais dinâmicas}

A utilização da expressão associada ao construto Capacidades Gerenciais Dinâmicas tem seu início no ano de 2003, por meio da publicação do trabalho intitulado "Corporate effects and dynamic managerial capabilities", de autoria Adner \& Helfat (2003). 0 artigo passou a ser referência sobre o tema, várias pesquisas foram realizadas e trabalhos publicados, tornando a teoria cada vez mais robusta. 0 Quadro 1, em sequência, elenca os diversos artigos sobre o tema. Logo depois, a análise geral realizada trará um panorama de como se encontram as discussões na atualidade (até o momento em que esse trabalho está sendo produzido), porém, considerando o número de artigos apresentados, não será possível analisálos individualmente, como na seção anterior.

Desde 2003, quando Adner e Helfat (2003) propuseram o construto, algumas outras teorias complementares foram surgindo, como é o caso das Capacidades Dinâmicas Individuais, propostas por Teece (2007), que também abarcam os gerentes. Por meio da leitura dos textos, é possível perceber fortes 
influências em relação à percepção, ao aproveitamento e à reconfiguração.

Quadro 1 - Títulos, autores e ano de publicação

\begin{tabular}{|c|c|c|}
\hline Título do artigo & Autor(es) & Ano \\
\hline Corporate effects and dynamic managerial capabilities & Adner \& Helfat & 2003 \\
\hline $\begin{array}{l}\text { Managerial discretion and internal alignment under regulatory constraints and } \\
\text { change }\end{array}$ & Peteraf \& Reed & 2007 \\
\hline $\begin{array}{l}\text { Cognition and renewal: comparing CEO and organizational effects on incumbent } \\
\text { adaptation to technical change }\end{array}$ & Eggers \& Kaplan & 2009 \\
\hline $\begin{array}{l}\text { Capabilities unveiled: the role of ordinary activities in the evolution of product } \\
\text { development processes }\end{array}$ & Salvato & 2009 \\
\hline $\begin{array}{l}\text { Contingencies within dynamic managerial capabilities: interdependent effects of } \\
\text { resource investment and deployment on firm performance }\end{array}$ & Sirmon \& Hitt & 2009 \\
\hline $\begin{array}{l}\text { Dynamic managerial capabilities and the multibusiness team: the role of epi- } \\
\text { sodic teams in executive leadership groups }\end{array}$ & Martin & 2011 \\
\hline $\begin{array}{l}\text { Executive decision making linking dynamic managerial capabilities to the re- } \\
\text { source portfolio and strategic outcomes }\end{array}$ & Beck \& Wiersema & 2013 \\
\hline $\begin{array}{l}\text { Dynamic managerial capabilities: configuration and orchestration of top execu- } \\
\text { tives' capabilities and the firm's dominant logic }\end{array}$ & Kor \& Mesko & 2013 \\
\hline $\begin{array}{l}\text { Founder-key leaders, group-level decision teams, and the international expan- } \\
\text { sion of business groups evidence from Taiwan }\end{array}$ & Lin & 2014 \\
\hline $\begin{array}{l}\text { Turning water into wine? Exploring the role of dynamic capabilities in early- } \\
\text { stage capitalization processes }\end{array}$ & Townsend \& Busenitz & 2015 \\
\hline $\begin{array}{l}\text { International opportunity recognition in international new ventures-a dynamic } \\
\text { managerial capabilities perspective }\end{array}$ & Andersson \& Evers & 2015 \\
\hline $\begin{array}{l}\text { Dynamic managerial capabilities: review and assessment of managerial impact } \\
\text { on strategic change }\end{array}$ & Helfat \& Martin & 2015 \\
\hline $\begin{array}{l}\text { Managerial cognitive capabilities and the microfoundations of dynamic capabili- } \\
\text { ties }\end{array}$ & Helfat \& Peteraf & 2015 \\
\hline $\begin{array}{l}\text { Examining a key corporate role: the influence of capital allocation competency } \\
\text { on business unit performance }\end{array}$ & $\begin{array}{l}\text { Arrfelt, Wiseman, } \\
\text { McNamara, \& Hult }\end{array}$ & 2015 \\
\hline $\begin{array}{l}\text { The impact of managerial cognition, human capital and social capital on strate- } \\
\text { gic entrepreneurship and firm performance: evidence from indonesian islamic } \\
\text { bank industry - ProQuest }\end{array}$ & Gusman \& Febrian & 2016 \\
\hline $\begin{array}{l}\text { Using information systems to sense opportunities for innovation: integrating } \\
\text { postadoptive use behaviors with the dynamic managerial capability perspective }\end{array}$ & $\begin{array}{l}\text { Roberts, Campbell, \& Vi- } \\
\text { jayasarathy }\end{array}$ & 2016 \\
\hline $\begin{array}{l}\text { MNE performance during a crisis: an evolutionary perspective on the role of dy- } \\
\text { namic managerial capabilities and industry context }\end{array}$ & Fainshmidt, Nair \& Mallon & 2017 \\
\hline $\begin{array}{l}\text { Individual dynamic managerial capabilities: influence over environmental and } \\
\text { social commitment under a gender perspective }\end{array}$ & $\begin{array}{l}\text { Buil-Fabrega, del Mar } \\
\text { Alonso-Almeida, \& Bagur- } \\
\text { Femenias }\end{array}$ & 2017 \\
\hline $\begin{array}{l}\text { Customer benefits and value creation in streaming services marketing: a mana- } \\
\text { gerial cognitive capability approach }\end{array}$ & Chen, Liu, \& Chiu & 2017 \\
\hline $\begin{array}{l}\text { Shedding light on sustainable development and stakeholder engagement: the } \\
\text { role of individual dynamic capabilities }\end{array}$ & $\begin{array}{l}\text { del Mar Alonso-Almeida, } \\
\text { Buil-Fabrega, Bagur-Fe- } \\
\text { menias, \& Pedro }\end{array}$ & 2017 \\
\hline $\begin{array}{l}\text { Heterogeneity in learning processes and the evolution of dynamic managerial } \\
\text { capabilities as a response of emergence of biosimilar market: evidence from the } \\
\text { indian pharmaceutical industry }\end{array}$ & Kale \& Huzair & 2017 \\
\hline $\begin{array}{l}\text { The impact of intellectual capital and knowledge flows on incremental and radi- } \\
\text { cal innovation: empirical findings from a transition economy of Vietnam }\end{array}$ & Duy Quoc & 2018 \\
\hline Executive capability for innovation: the irish seaports sector & $\begin{array}{l}\text { Kearney, Harrington, \& } \\
\text { Kelliher }\end{array}$ & 2018 \\
\hline $\begin{array}{l}\text { Capabilities in business relationships and networks: research recommendations } \\
\text { and directions }\end{array}$ & $\begin{array}{l}\text { Forkmann, Henneberg, \& } \\
\text { Mitrega }\end{array}$ & 2018 \\
\hline $\begin{array}{l}\text { A relational perspective of the microfoundations of dynamic managerial capa- } \\
\text { bilities and transactive memory systems }\end{array}$ & Martin \& Bachrach & 2018 \\
\hline Digital transformation by SME entrepreneurs: a capability perspective & Li, Su, Zhang, \& Mao & 2018 \\
\hline $\begin{array}{l}\text { Firm functions and the nature of competitive advantage in internationalizing } \\
\text { SMEs }\end{array}$ & $\begin{array}{l}\text { Haapanen, Hurmelinna- } \\
\text { Laukkanen, \& Hermes }\end{array}$ & 2018 \\
\hline $\begin{array}{l}\text { Stakeholder pressure and its effect on environmental proactiveness and firm } \\
\text { performance: the mediating role of dynamic managerial capabilities }\end{array}$ & $\begin{array}{l}\text { Pusparini, Soetjipto, Rach- } \\
\text { mawati, \& Sudhartio }\end{array}$ & 2018 \\
\hline $\begin{array}{l}\text { Resource orchestration in start-ups: synchronizing human capital investment, } \\
\text { leveraging strategy, and founder start-up experience }\end{array}$ & Symeonidou \& Nicolaou & 2018 \\
\hline
\end{tabular}




\begin{tabular}{|l|l|c|}
\hline \multicolumn{1}{|c|}{ Título do artigo } & \multicolumn{1}{|c|}{ Autor(es) } & Ano \\
\hline $\begin{array}{l}\text { A study of IS assets, IS ambidexterity, and IS alignment: the dynamic managerial } \\
\text { capability perspective }\end{array}$ & Tai, Wang, \& Yeh & 2019 \\
\hline $\begin{array}{l}\text { The antecedents and the outcomes of foreign market knowledge accumulation - } \\
\text { the dynamic managerial capability perspective }\end{array}$ & $\begin{array}{l}\text { Mostafiz, Sambasivan, \& } \\
\text { Goh }\end{array}$ & 2019 \\
\hline $\begin{array}{l}\text { The mediating role of dynamic managerial capabilities: the interplay between } \\
\text { dominant logic and small- and medium-sized enterprises performance in china }\end{array}$ & $\begin{array}{l}\text { Khan, Atlas, Zhang, \& } \\
\text { Khan }\end{array}$ & 2019 \\
\hline $\begin{array}{l}\text { The impact of dominant logic and competitive intensity on SMEs performance: a } \\
\text { case from china }\end{array}$ & $\begin{array}{l}\text { Khan, Zhang, Atlas, \& } \\
\text { Khan }\end{array}$ & 2019 \\
\hline $\begin{array}{l}\text { Do boards of directors foster strategic change? A dynamic managerial capabili- } \\
\text { ties perspective }\end{array}$ & Åberg \& Shen & 2019 \\
\hline $\begin{array}{l}\text { Business, society, and the need for stewardship orientation } \\
\text { Dynamic managerial capabilities: scale development and validation }\end{array}$ & $\begin{array}{l}\text { Nijhof, Schaveling, \& } \\
\text { Zalesky }\end{array}$ & 2019 \\
\hline $\begin{array}{l}\text { Impacts of dynamic managerial capability and international opportunity identi- } \\
\text { fication on firm performance }\end{array}$ & $\begin{array}{l}\text { Mostafiz, Sambasivan, \& } \\
\text { Goh }\end{array}$ & 2019 \\
\hline $\begin{array}{l}\text { Exploring the affective underpinnings of dynamic managerial capabilities: how } \\
\text { managers' emotion regulation behaviors mobilize resources for their firms }\end{array}$ & Huy \& Zott & 2019 \\
\hline $\begin{array}{l}\text { Can board leadership contribute to board dynamic managerial capabilities? An } \\
\text { empirical exploration among norwegian firms }\end{array}$ & Åberg \& Shen & 2019 \\
\hline
\end{tabular}

Notas. Fonte: Elaborado pelo autor

No Quadro 1, encontram-se pesquisas que utilizam diversos contextos, como o de tecnologia (Tai, Wang, \& Yeh, 2019), aspectos relacionados à capacidade decisória dos gerentes (Beck \& Wiersema, 2013; Kearney, Harrington, \& Kelliher, 2018; Forkmann, Henneberg, \& Mitrega, 2018), às capacidades gerenciais dinâmicas com a internacionalização (Peteraf \& Reed, 2007; Lin, 2014; Andersson \& Evers, 2015; Haapanen, Hurmelinna-Laukkanen, \& Hermes, 2018, Mostafiz, 2019), além de outras perspectivas que são abordadas.

Helfat e Martin (2015) publicaram um estudo de revisão e avaliação do construto Capacidades Gerenciais Dinâmicas, por meio de um levantamento em relação a como tem sido abordado o tema, as publicações e estudos realizados. Segundo os autores, um fato chama a atenção: até 2015, nenhuma pesquisa teve como escopo o estudo dos três atributos de forma conjunta (capital social gerencial, capital humano gerencial e cognição gerencial).

Já na análise realizada neste trabalho, em um momento mais atual, foi possível perceber que, apenas a partir de 2018, houve uma evolução nesse aspecto, aumentando a frequência de estudos que abordam tais atributos. Mesmo assim, ainda é possível encontrar, atualmente, diversos artigos que não abordam todos eles, ou, quando o fazem, é de maneira genérica, ou seja, utilizam-se da teoria das Capacidade Gerenciais Dinâmicas, mas não objetivam aprofundar o construto e suas nuances, como é possível observar na Tabela 6.

Tabela 6 - Abordagem dos atributos das Capacidades Gerencias Dinâmicas nos artigos analisados

\begin{tabular}{|l|c|c|}
\hline Abordagem dos atributos & Frequência & Percentual \\
\hline Abordagem genérica & 23 & $58,97 \%$ \\
\hline Todos os atributos & 9 & $23,08 \%$ \\
\hline Cognição Gerencial & 3 & $7,69 \%$ \\
\hline Capital Humano Gerencial & 2 & $5,14 \%$ \\
\hline Capital Social Gerencial & 1 & $2,56 \%$ \\
\hline Capital Humano Gerencial e Cognição Gerencial & 1 & $2,56 \%$ \\
\hline Total & 39 & $100 \%$ \\
\hline
\end{tabular}

Notas. Fonte: Elaborado pelo autor

Como é possível observar nos dados apresentados, 58,97\% dos artigos analisados abordam os atributos do construto Capacidade Gerencial Dinâmica de maneira genérica, ou seja, são trabalhos que citam os conceitos, porém não os aprofundam, explorando aspectos mais superficiais.

Na revisão da literatura dos autores Helfat e Martin (2015), há uma crítica em relação à quantidade de estudos que versem sobre todos os atributos do construto. É possível perceber, entretanto, que $23,08 \%$ da amostra abordam simultaneamente os três atributos propostos por Adner e Helfat (2003). 
Analisando os trabalhos identificados que tratam dos três atributos (Andersson \& Evers, 2015; Gusman \& Febrian, 2016; Martin \& Bachrach, 2018; Li, Su, Zhang, \& Mao, 2018; Khan, Atlas, Zhang, \& Khan, 2019; Åberg \& Shen, 2019; Åberg \& Shen, 2020; Correa, Bueno, Kato, \& De Oliveira Silva, 2019; Mostafiz, 2019), percebe-se que estes buscam ampliar o conhecimento em relação à aplicabilidade dentro de um contexto prático e estratégico empresarial. Ainda assim, não é possível identificar, porém, trabalhos que aprofundem as explicações sobre como direcionar o desenvolvimento das Capacidades Gerenciais Dinâmicas.

A partir da leitura e análise dos artigos indicados na relação de trabalhos apresentados no Quadro 1, é possível perceber que o construto Capacidade Gerenciais Dinâmicas possui vários estudos empíricos (que consequentemente validam, comprovam ou complementam conhecimentos e nuances relacionadas ao tema), porém são, em sua maioria, teóricos, como é possível observar na Tabela .

Tabela 7 - Tipos de Pesquisas utilizados nos estudos sobre Capacidades Gerenciais Dinâmicas

\begin{tabular}{|l|c|c|}
\hline Tipos de Pesquisa & Frequência & Percentual \\
\hline Empírico & 19 & $48,72 \%$ \\
\hline Teórico & 11 & $28,21 \%$ \\
\hline Teórico - Empírico & 9 & $23,07 \%$ \\
\hline Total & $\mathbf{3 9}$ & $\mathbf{1 0 0 \%}$ \\
\hline
\end{tabular}

Notas. Fonte: Elaborado pelo autor

Identificaram-se, ainda, vários trabalhos do tipo teórico - 28,21\% - que objetivam complementar ou dar uma nova visão ao construto, o que de certa maneira preocupa aqueles que estão construindo uma pesquisa baseada na essência do construto, seus atributos. Já os artigos empíricos, estes permitem compreender como são aplicados na prática e como estão sendo estudados em determinados contextos e representam 48,72\% dos artigos analisados. Existem, porém, aqueles que, de modo empírico, trazem novas teorias e/ou complementações ao construto já existente, o que representa $23,07 \%$ da amostra.

Analisando o acesso aos dados e às estratégias de pesquisa, identificou-se que, apesar da grande quantidade de trabalhos empíricos, que no total representam $71,79 \%$ da amostra, é possível perceber que as informações analisadas são obtidas por meio de bancos de dados existentes e disponíveis para o público e Surveys, apesar de também existir uma quantidade significativa de Estudos de Casos, Revisão da Literatura e outros, como é possível observar na Tabela .

Tabela 8 - Métodos de Pesquisas utilizados nos estudos sobre Capacidades Gerenciais Dinâmicas

\begin{tabular}{|l|c|c|}
\hline Método de pesquisa & Frequência & Percentual \\
\hline Survey & 13 & $33,34 \%$ \\
\hline Análise de dados & 8 & $20,51 \%$ \\
\hline Estudo de caso & 8 & $20,51 \%$ \\
\hline Ensaio Teórico & 5 & $12,82 \%$ \\
\hline Revisão da literatura & 5 & $12,82 \%$ \\
\hline Total & $\mathbf{3 9}$ & $\mathbf{1 0 0 \%}$ \\
\hline
\end{tabular}

Notas. Fonte: Elaborado pelo autor

O método de pesquisa Survey apresentou maior frequência, o que está alinhado ao número de pesquisas empíricas encontradas. Chama-nos a atenção a quantidade de trabalhos que se utilizam do método de análise de dados, ou seja, que buscam em plataforma ou base de dados as informações disponíveis para realizar a análise. Tal método é considerado válido, contudo não há o contato do pesquisador com o campo, fugindo, assim, do conhecimento real e empírico do ambiente de pesquisa.

Os métodos de pesquisa estão alinhados diretamente com as abordagens utilizadas nos trabalhos analisados, como pode ser observado na Tabela .

Tabela 9 - Abordagens utilizadas nos estudos sobre Capacidades Gerenciais Dinâmicas

\begin{tabular}{|l|c|c|}
\hline Abordagem & Frequência & Percentual \\
\hline Quantitativa & 21 & $53,85 \%$ \\
\hline Qualitativa & 10 & $25,64 \%$ \\
\hline Ensaio teórico & 5 & 12,82 \\
\hline Qualitativa e Quantitativa & 3 & $7,69 \%$ \\
\hline Total & $\mathbf{3 9}$ & $\mathbf{1 0 0 \%}$ \\
\hline
\end{tabular}

Notas. Fonte: Elaborado pelo autor 
Constata-se uma predominância internacional dos pesquisadores na utilização de métodos quantitativos, com análises estatísticas que permitem alcançar critérios de validade e confiabilidade de maneira mais objetiva, além do fato que é possível utilizar abordagens multiparadigmáticas nos estudos. É deixada de lado, contudo, a subjetividade inerente ao pesquisador.

Utilizando-se da subjetividade e analisando de maneira mais qualitativa, após a leitura dos artigos selecionados, foi possível observar a evolução das teorias e estudos relacionados ao gerente, o que se deve ao trabalho de Adner e Helfat (2003), que entenderam, sob uma nova ótica, o papel desse executivo no desenvolvimento estratégico da organização. Os estudos voltados para Capacidades Dinâmicas estão, entretanto, mais centrados em uma perspectiva macro-organizacional, ou seja, ainda não há uma pesquisa que objetive compreender os atributos (capital humano gerencial, capital social gerencial e cognição gerencial) de maneira mais profunda, cogitando-se reflexões ou questionamentos, por exemplo: Como alavancar tais atributos no profissional? Como são desenvolvidas as Capacidades Gerenciais Dinâmicas? Quais ambientes podem fomentar o desenvolvimento das CGDs? Entre outras questões.

Considerando os gaps existentes e percebidos nos trabalhos, é possível afirmar que ainda existem diversas áreas que precisam ser investigadas, como em certos tipos de formação de empresas como cluster, join ventures, aglomerados industriais, que representam uma lacuna de pesquisa. Da mesma forma, ainda não existem trabalhos suficientes que contemplem todos os atributos de maneira explicativa, ou seja, que tenha um foco voltado especificamente para o desenvolvimento dos atributos, além de trabalhos que deixem explícito como são desenvolvidas as capacidades gerenciais dinâmicas.

\subsection{Da teoria à prática}

A revisão sistemática da literatura revelou que, apesar dos avanços nas pesquisas sobre Capacidades Dinâmicas, a perspectiva gerencial ainda representa uma lacuna teórica a ser preenchida e que, para compreender uma determinada temática, é preciso identificar o seu estado da arte. Do ponto de vista teórico, os resultados deste estudo tornam-se relevantes para o conhecimento dos construtos Capacidades Dinâmicas e Capacidades Gerenciais Dinâmicas, uma vez que podem indicar, para os leitores, como está o desenvolvimento de pesquisas em relação ao papel do gerente nesses construtos. A compreensão do fenômeno, do ponto de vista teórico, também tem uma implicação para a prática gerencial, ampliando a percepção de como a ação gerencial atua no desenvolvimento de capacidades dinâmicas.

Os resultados deste trabalho podem exercer influência no meio acadêmico, visto que o desenvolvimento científico do tema só é possível por meio da pesquisa científica que identifique fatores determinantes e mediadores do desenvolvimento de capacidades gerenciais dinâmicas, além de modelos ou estruturas de referências que permitam a proposição de perspectivas teóricas com repercussões para a prática.

Em ambientes complexos e dinâmicos, as empresas buscam adaptar seus recursos, internos e externos, objetivando o melhor aproveitamento das oportunidades e, muitas vezes, o(s) agente(s) central(is) desse processo é(são) o(s) gerente(s). Compreender quais características (formação, social ou cognitiva) podem potencializar o desenvolvimento de competências e as capacidades gerenciais dinâmicas são a chave para analisar o comportamento de uma organização ou de um aglomerado de empresas, no desenvolvimento das Capacidades Dinâmicas, de maneira estratégica.

\section{Conclusão}

Este estudo possibilitou ampliar a compreensão sobre o desenvolvimento das pesquisas relacionadas à Capacidades Dinâmicas, com ênfase no papel do gerente. Percebeu-se que a existência de um construto específico e focado na atuação desse profissional fez com que houvesse mais pesquisas relacionadas. Ainda assim, é possível afirmar, contudo, que, para aprofundar os conceitos relacionados às Capacidades Dinâmicas Gerenciais, faz-se necessária a realização de pesquisas que ampliem o conhecimento de como pode ser impulsionado o desenvolvimento dos atributos gerenciais (capital humano, capital social e cognição gerencial).

Observou-se que poucas pesquisas focaram no papel do gerente quando se utiliza uma lente mais ampla da teoria das Capacidades Dinâmicas. A quantidade de pesquisas é maior, porém, quando utilizado o construto Capacidades Gerenciais Dinâmicas. 0 número de pesquisas empíricas que busque no campo respostas (e não por meio de base de dados) ainda é considerado baixo, o que aponta para a 
necessidade do desenvolvimento de mais pesquisas desse tipo, para que se possa melhorar e consolidar os atuais achados.

Com este trabalho, é possível ter uma melhor visão de como estão direcionadas as pesquisas sobre o gerente no âmbito das Capacidades Dinâmicas, apotando para a maior necessidade de estudos que tenham como foco os indivíduos, em especial os gerentes, além de aspectos ambientais que possam fomentar o desenvolvimento de competências e atributos gerenciais.

Entende-se a necessidade de pesquisas sobre Capacidades Gerenciais Dinâmicas sobre ambientes de aglomerações industriais, como clusters, a troca de informações entre empresas e o aprendizado profissional; o impacto de áreas em que há concentração educacional e a absorção de profissionais gestores; programas de desenvolvimento profissionais internos voltados para capacitação e aprendizagem são estudos que podem ser desenvolvidos.

Este artigo apresenta lacunas que não comprometem sua qualidade, mas que podem apresentarse como limitações. Sobre essas lacunas, podemos dizer que, em primeiro, entende-se que, pelo fato de a pesquisa ter sido realizada em apenas uma base de dados (embora seja mais relevante e com maior acervo), é possível que em outras bases existam trabalhos que complementem ou modifiquem nossos atuais resultados. Em segundo, as análises realizadas poderiam alcançar maior nível de profundidade e detalhes, porém, tornariam a leitura excessivamente longa, logo exaustiva. Por fim, embora não tenha sido possível analisar um dos artigos do corpus na íntegra, por sua inacessibilidade, acredita-se que tal fato não modificaria os resultados obtidos.

Apesar das lacunas, este trabalho possui contribuições acadêmicas por permitir uma investigação do cenário de pesquisa sobre o gerente e sua relação com as Capacidades Dinâmicas e por elencar trabalhos que podem ser fontes de leitura para aqueles que objetivam aprofundar-se na temática.

De maneira prática e profissional, os artigos empíricos referenciados nesse trabalho podem ser uma rica fonte de pesquisa para o desenvolvimento de estratégias organizacionais de internacionalização e desenvolvimento cognitivos e sociais. Por meio de pesquisas sugeridas neste trabalho, quiçá em um futuro próximo, obter-se-á um maior conhecimento de como aprimorar os atributos gerenciais para alavancar o desenvolvimento de Capacidades Gerenciais Dinâmicas.

\section{Nota de Agradecimento:}

Este artigo é parte de uma pesquisa que recebeu apoio financeiro da Coordenação de Aperfeiçoamento de Pessoal de Nível Superior (CAPES)

\section{Referências}

Åberg, C., \& Shen, W. (2020). Can board leadership contribute to board dynamic managerial capabilities? An empirical exploration among Norwegian firms. Journal of Management and Governance, 24(1), 169197.

Åberg, C., \& Torchia, M. (2019). Do boards of directors foster strategic change? A dynamic managerial capabilities perspective. Journal of Management and Governance, 1-30.

Adner, R., \& Helfat, C. E. (2003). Corporate effects and dynamic managerial capabilities. Strategic management journal, 24(10), 1011-1025.

Akrofi, S. (2016). Evaluating the effects of executive learning and development on organisational performance: implications for developing senior manager and executive capabilities. International Journal of Training and Development, 20(3), 177-199.

Andersson, S., \& Evers, N. (2015). International opportunity recognition in international new venturesa dynamic managerial capabilities perspective. Journal of International Entrepreneurship, 13(3), 260276.

Ansari, R., Barati, A., \& Sharabiani, A. A. A. (2016). The role of dynamic capability in intellectual capital and innovative performance. International Journal of Innovation and Learning, 20(1), 47-67.

Araújo, C. A. (2006). Bibliometria: evolução histórica e questões atuais. Em questão, 12(1), 11-32.

Arrfelt, M., Wiseman, R. M., McNamara, G., \& Hult, G. T. M. (2015). Examining a key corporate role: The influence of capital allocation competency on business unit performance. Strategic Management Journal, 36(7), 1017-1034.

Augier, M., \& Teece, D. J. (2009). Dynamic capabilities and the role of managers in business strategy and economic performance. Organization science, 20(2), 410-421. 
Beck, J. B., \& Wiersema, M. F. (2013). Executive decision making: Linking dynamic managerial capabilities to the resource portfolio and strategic outcomes. Journal of Leadership \& Organizational Studies, 20(4), 408-419.

Buil-Fabregà, M., del Mar Alonso-Almeida, M., \& Bagur-Femenías, L. (2017). Individual dynamic managerial capabilities: Influence over environmental and social commitment under a gender perspective. Journal of cleaner production, 151, 371-379.

Carpenter, M. A., Sanders, W. G., \& Gregersen, H. B. (2001). Bundling human capital with organizational context: The impact of international assignment experience on multinational firm performance and CEO pay. Academy of management journal, 44(3), 493-511.

Chang, J., Bai, X., \& Li, J. J. (2015). The influence of leadership on product and process innovations in China: The contingent role of knowledge acquisition capability. Industrial Marketing Management, 50, 18-29.

Chen, Y. M., Liu, H. H., \& Chiu, Y. C. (2017). Customer benefits and value creation in streaming services marketing: a managerial cognitive capability approach. Psychology \& Marketing, 34(12), 1101-1108.

Conforto, E. C., Amaral, D. C., \& Silva, S. D. (2011). Roteiro para revisão bibliográfica sistemática: aplicação no desenvolvimento de produtos e gerenciamento de projetos. Trabalho apresentado, $8^{\text {o }}$ Congresso Brasileiro de Gestão de Desenvolvimento de Produto - CBGDP 2011, 2011, Porto Alegre-RS.

Corrêa, R. O., Bueno, E. V., Kato, H. T., \& Silva, L. M. D. O. (2019). Dynamic managerial capabilities: Scale development and validation. Managerial and Decision Economics, 40(1), 3-15.

del Mar Alonso-Almeida, M., Buil-Fabregà, M., Bagur-Femenías, L., \& Aznar-Alarcón, J. P. (2017) Shedding light on sustainable development and stakeholder engagement: The role of individual dynamic capabilities. Sustainable Development, 25(6), 625-638.

Eggers, J. P., \& Kaplan, S. (2009). Cognition and renewal: Comparing CEO and organizational effects on incumbent adaptation to technical change. Organization Science, 20(2), 461-477.

Eisenhardt, K. M., \& Martin, J. A. (2000). Dynamic capabilities: what are they?. Strategic management journal, 21(10-11), 1105-1121.

Fainshmidt, S., Nair, A., \& Mallon, M. R. (2017). MNE performance during a crisis: An evolutionary perspective on the role of dynamic managerial capabilities and industry context. International Business Review, 26(6), 1088-1099.

Fernández-Mesa, A., Iborra, M., \& Safón, V. (2013). CEO-TMT interaction: do tenure and age affect ambidexterity dynamism?. European Journal of International Management, 7(1), 31-55.

Forkmann, S., Henneberg, S. C., \& Mitrega, M. (2018). Capabilities in business relationships and networks: Research recommendations and directions. Industrial Marketing Management, 74, 4-26.

Gusman, Y., \& Febrian, E. (2016). The impact of managerial cognition, human capital and social capital on strategic entrepreneurship and firm performance: Evidence from Indonesian Islamic Bank Industry. Academy of Strategic Management Journal, 15, 82-94.

Haapanen, L., Hurmelinna-Laukkanen, P., \& Hermes, J. (2018). Firm functions and the nature of competitive advantage in internationalizing SMEs. International Journal of Innovation Management, 22(03), 1850022.

Helfat, C. E., \& Martin, J. A. (2015). Dynamic managerial capabilities: Review and assessment of managerial impact on strategic change. Journal of management, 41(5), 1281-1312.

Helfat, C. E., \& Peteraf, M. A. (2015). Managerial cognitive capabilities and the microfoundations of dynamic capabilities. Strategic Management Journal, 36(6), 831-850.

Huy, Q., \& Zott, C. (2019). Exploring the affective underpinnings of dynamic managerial capabilities: How managers' emotion regulation behaviors mobilize resources for their firms. Strategic Management Journal, 40(1), 28-54.

Kale, D., \& Huzair, F. (2017). Heterogeneity in learning processes and the evolution of dynamic managerial capabilities as a response of emergence of biosimilar market: evidence from the Indian pharmaceutical industry. Technology Analysis \& Strategic Management, 29(3), 300-312.

Kearney, A., Harrington, D., \& Kelliher, F. (2018). Executive capability for innovation: the Irish seaports sector. European Journal of Training and Development, 42(5/6), 342-361.

Khan, K. U., Atlas, F., Xuehe, Z., Khan, F., \& Khan, S. (2019). The mediating role of dynamic managerial capabilities: The interplay between dominant logic and small-and medium-sized enterprises performance in China. Journal of Public Affairs, e2002. 
Khan, K. U., Xuehe, Z., Atlas, F., \& Khan, F. (2019). The impact of dominant logic and competitive intensity on SMEs performance: A case from China. Journal of Innovation \& Knowledge, 4(1), 1-11.

King, W. R., \& He, J. (2005). Understanding the role and methods of meta-analysis in IS research. Communications of the Association for Information Systems, 16(1), 665-686.

Kitchenham, B., Pretorius, R., Budgen, D., Brereton, O. P., Turner, M., Niazi, M., \& Linkman, S. (2010). Systematic literature reviews in software engineering-a tertiary study. Information and software technology, 52(8), 792-805.

Kor, Y. Y., \& Mesko, A. (2013). Dynamic managerial capabilities: Configuration and orchestration of top executives' capabilities and the firm's dominant logic. Strategic management journal, 34(2), 233-244.

Lecler, C. J., \& Kinghorn, J. (2014). Dynamic capabilities, expert and entrepreneurial learning. South African Journal of Business Management, 45(2), 65-81.

Li, L., Su, F., Zhang, W., \& Mao, J. Y. (2018). Digital transformation by SME entrepreneurs: A capability perspective. Information Systems Journal, 28(6), 1129-1157.

Lin, W. T. (2014). Founder-key leaders, group-level decision teams, and the international expansion of business groups. International Marketing Review, 31(2), 129-154.

Martin, J. A. (2011). Dynamic managerial capabilities and the multibusiness team: The role of episodic teams in executive leadership groups. Organization Science, 22(1), 118-140.

Martin, J. A., \& Bachrach, D. G. (2018). A relational perspective of the microfoundations of dynamic managerial capabilities and transactive memory systems. Industrial Marketing Management, 74, 27-38.

Mostafiz, M. I., Sambasivan, M., \& Goh, S. K. (2019a). Impacts of dynamic managerial capability and international opportunity identification on firm performance. Multinational Business Review, 27(4), 339363.

Mostafiz, M. I., Sambasivan, M., \& Goh, S. K. (2019b). The antecedents and the outcomes of foreign market knowledge accumulation-the dynamic managerial capability perspective. Journal of Business \& Industrial Marketing, 34(4), 902-920.

Narayanan, V. K., Colwell, K., \& Douglas, F. L. (2009). Building organizational and scientific platforms in the pharmaceutical industry: A process perspective on the development of dynamic capabilities. British Journal of Management, 20, S25-S40.

Nguyen, D. Q. (2018). The impact of intellectual capital and knowledge flows on incremental and radical innovation. Asia-Pacific Journal of Business Administration, 10(2/3), 149-170.

Nijhof, A., Schaveling, J., \& Zalesky, N. (2019). Business, society, and the need for stewardship orientation. Journal of Organizational Change Management, 32(1), 145-163.

O'Reilly III, C. A., \& Tushman, M. L. (2011). Organizational ambidexterity in action: How managers explore and exploit. California management review, 53(4), 5-22.

Penrose, E., \& Penrose, E. T. (2009). The Theory of the Growth of the Firm. Oxford University Press.

Peteraf, M., \& Reed, R. (2007). Managerial discretion and internal alignment under regulatory constraints and change. Strategic Management Journal, 28(11), 1089-1112.

Pusparini, E. S., Soetjipto, B. W., Rachmawati, R., \& Sudhartio, L. (2018). Stakeholder Pressure and Its Effect on Environmental Proactiveness and Firm Performance: The Mediating Role of Dynamic Managerial Capabilities. Pertanika Journal of Social Sciences and Humanities, 26(August), 197-212.

Ramachandran, I. (2018). Triggering absorptive capacity in organizations: CEO succession as a knowledge enabler. Journal of Knowledge Management, 22(8), 1844-1864.

Roberts, N., Campbell, D. E., \& Vijayasarathy, L. R. (2016). Using information systems to sense opportunities for innovation: Integrating postadoptive use behaviors with the dynamic managerial capability perspective. Journal of Management Information Systems, 33(1), 45-69.

Salvato, C. (2009). Capabilities unveiled: The role of ordinary activities in the evolution of product development processes. Organization Science, 20(2), 384-409.

Singh, B., \& Rao, M. K. (2016). Effect of intellectual capital on dynamic capabilities. Journal of Organizational Change Management, 29(2),129-149.

Sirmon, D. G., \& Hitt, M. A. (2009). Contingencies within dynamic managerial capabilities: Interdependent effects of resource investment and deployment on firm performance. Strategic Management Journal, 30(13), 1375-1394.

Symeonidou, N., \& Nicolaou, N. (2018). Resource orchestration in start-ups: Synchronizing human cap- 
ital investment, leveraging strategy, and founder start-up experience. Strategic Entrepreneurship Journal, 12(2), 194-218.

Tai, J. C., Wang, E. T., \& Yeh, H. Y. (2019). A study of IS assets, IS ambidexterity, and IS alignment: the dynamic managerial capability perspective. Information \& Management, 56(1), 55-69.

Teece, D. J. (2007). Explicating dynamic capabilities: the nature and microfoundations of (sustainable) enterprise performance. Strategic management journal, 28(13), 1319-1350.

Teece, D. J., Pisano, G., \& Shuen, A. (1997). Dynamic capabilities and strategic management. Strategic management journal, 18(7), 509-533.

Townsend, D. M., \& Busenitz, L. W. (2015). Turning water into wine? Exploring the role of dynamic capabilities in early-stage capitalization processes. Journal of Business Venturing, 30(2), 292-306.

Wernerfelt, B. (1984). A resource-based view of the firm. Strategic management journal, 5(2), 171-180. Witcher, B. J., Chau, V. S., \& Harding, P. (2008). Dynamic capabilities: top executive audits and hoshin kanri at Nissan South Africa. International Journal of Operations \& Production Management, 28(6), 540561.

Zollo, M., \& Winter, S. G. (2002). Deliberate learning and the evolution of dynamic capabilities. Organization science, 13(3), 339-351. 